\title{
An indicator for month-trading of stocks
}

\author{
You Beng Koh ${ }^{1 *}$, Yew Seong $\mathrm{Ng}^{2}$, and Ah Hin Pooi $^{3}$ \\ ${ }^{1}$ Institute of Mathematical Sciences, Faculty of Science, Universiti Malaya, 50603, Kuala Lumpur, \\ Malaysia \\ ${ }^{2}$ Metrology Service Center PLT, 40460, Selangor, Malaysia \\ ${ }^{3}$ Centre for Mathematical Sciences, Universiti Tunku Abdul Rahman, 43000, Selangor, Malaysia
}

\begin{abstract}
Some individual investors who find day-trading of stocks incompatible with their lifestyle might want to adopt month-trading instead. In this paper, we use the historical monthly share prices to construct an indicator to guide us in trading a portfolio of two stocks in the Malaysian stock market on a monthly basis. Apart from helping us in the selection of the two stocks, the indicator also provides guidance on the choice of the weight of each stock in the portfolio and the determination of the time to invest in the portfolio.
\end{abstract}

\section{Introduction}

The patterns of price history of a financial instrument sometimes provide indications of the future behaviour of prices. A number of indicators have been proposed to analyse the price patterns of stocks. The common indicators include the moving average, the moving average convergence divergence (MACD) [1], the relative strength index (RSI) [2] and the onbalance volume (OBV) [3].

Trading strategies may also be formulated by using objective mathematical models for the asset prices. In order to increase the predictive power of the models, some authors incorporated macroeconomic variables in the models $[4,5,6,7]$. Pesaran and Timmermann [4] introduced variable selection procedure to identify a base set of predictors at each point in time. Kyereboah-Coleman and Agyire-Tettey [5] examined the impact of macroeconomic variables on the performance of Ghana stock market. Cakmakli and van Dijk [6] used dynamic factor models on a large set of pre-selecting macroeconomic variables for predicting monthly US excess stock returns over the period January 1975-December 2014. Liu and Kemp [7] combined the forward sequential variable selection algorithm and information criteria to identify the macroeconomic variables for forecasting the excess return on US oil and gas industry.

The relationship between the macroeconomic variables and stock market in Malaysia has been studied by various researchers $[8,9,10,11,12]$. Thaker et al. [8] reported inflation, money supply and exchange rate significantly affect the stock market. Rahman et al. [9] showed the evidence that monetary policies variables and domestic supply factors have significant long run effect on the stock market. Zakaria and Shasuddin [10] found that the inflation rate and interest rate have a relationship with the stock market. Bekhet and

*Corresponding author: kohyoubeng@um.edu.my 
Mugableh [11] showed the equilibrium relationship between macroeconomic variables and stock market index. Recently, Nasser et al. [12] concluded that the inflation, interest rates and exchange rate have a negative correlation relationship while industrial production has a positive relationship with the index in the ASEAN region.

In this paper, we offer a technique to construct an indicator to guide investors in trading a portfolio of two Malaysian stocks on a monthly basis. Several factors are used in the model for predicting the prices of two selected stocks in the next $k$-th $(k=1,2)$ month. These factors include

(1) the present month values of a small number, $n_{L}$, of latent variables derived from a set of 17 selected monthly Malaysian macroeconomic variables,

(2) the present month value of the trading volume and composite index of the Malaysian stock market, and

(3) the present month values of the prices $S_{1}$ and $S_{2}$ and trading volumes $V_{1}$ and $V_{2}$ of two selected stocks.

Instead of using the original macroeconomic variables directly, the latent variables in item (1) above are used. The aim is to use a small number of variables to extract the explanatory power of a large number of macroeconomic variables.

We consider a portfolio which consists of $w_{i}$ units of stock $i, i=1,2$ with $w_{1}+w_{2}=1$. The present month's price of the portfolio is then given by

$$
S=S_{1} w_{1}+S_{2} w_{2}
$$

Let $S_{i}^{(k)}$ be the price of stock $i$ in the next $k$-th month, $i=1,2 ; k=1,2$, and $S^{(k)}=$ $S_{1}^{(k)} w_{1}+S_{2}^{(k)} w_{2}$ is the price of the portfolio in the next $k$-th month. The gain in the next $k$ th month (before taking into account the interest rate and other expenses) will be

$$
g^{(k)}=\left(S^{(k)}-\text { observed value of } S\right), \quad k=1,2 .
$$

The large value of the probability that the gain in next month is positive has been used as an indicator for trading the portfolio in [13]. In this paper, we wish to find out whether the large value of the probability that the gain in the second month in the future is positive is a better indicator for trading the portfolio. The numerical results show that there exist pairs of Malaysian stocks and the corresponding weights such that the actual gain next month tends to be positive when the probability of positive gain in the second month in the future is large. Furthermore, it is found that when the actual gain next month is regressed on the probability of positive gain in the second month, the slope of the regression tends to be larger than that in the case when the probability of positive gain in next month is used.

The layout of the paper is as follows. In Section 2, we introduced the multivariate powernormal (MPN) distribution given in [14]. In Section 3, the technique based on MPN distribution is used to find a conditional distribution for the value of the portfolio in the next $k(k=1,2)$ month in the future when the present month values of the $n_{L}+6$ factors listed in items (1)-(3) above are given. From the conditional distribution, we find the probability of getting positive gain $k$ months later. In Section 4, various pairs of the Malaysian stocks are chosen and for each chosen pair and the assigned weights $w_{1}$ and $w_{2}$, the actual gain next month is regressed on the probability of getting positive gain $k$ months later. Section 5 concludes the paper.

\section{Multivariate power-normal distribution}

Let $\boldsymbol{y}$ be a vector consisting of $k$ correlated random variables. The vector $\boldsymbol{y}$ is said to have a $k$-dimensional MPN distribution ([14]) with parameters $\boldsymbol{\mu}, \boldsymbol{H}, \lambda_{i}^{+}, \lambda_{i}^{-}$, and $\sigma_{i}$ for $i=1,2, \ldots, k$ if

$$
\boldsymbol{y}=\boldsymbol{\mu}+\boldsymbol{H} \boldsymbol{\varepsilon}
$$

where $\mu=\mathbb{E}[\boldsymbol{y}], \boldsymbol{H}$ is an orthogonal matrix, $\varepsilon_{1}, \varepsilon_{2}, \ldots, \varepsilon_{k}$ are independent, 
$\sigma_{i}>0$ is a constant,

$$
\varepsilon_{i}=\frac{\sigma_{i}\left[\tilde{\varepsilon}_{i}-\mathbb{E}\left[\tilde{\varepsilon}_{i}\right]\right]}{\sqrt{\operatorname{var}\left(\tilde{\varepsilon}_{i}\right)}}, \quad i=1,2, \ldots, k,
$$

$$
\tilde{\varepsilon}_{i}=\psi\left(\lambda_{i}^{+}, \lambda_{i}^{-}, z_{i}\right)=\left\{\begin{array}{cl}
\frac{\left[\left(z_{i}+1\right)^{\lambda_{i}^{+}}-1\right]}{\lambda_{i}^{+}}, & \left(z \geq 0, \lambda_{i}^{+} \neq 0\right) \\
-\frac{\left[\left(-z_{i}+1\right)^{\lambda_{i}^{-}}-1\right]}{\lambda_{i}^{-}}, & \left(z \geq 0, \lambda_{i}^{+}=0\right), i=1,2, \ldots k, \\
-\log \left(-z_{i}+1\right), & \left(z<0, \lambda_{i}^{-} \neq 0\right)
\end{array}\right.
$$

and $z_{i}, i=1,2, \ldots, k$ are independent standard normal random variables. The variable $\tilde{\varepsilon}_{i}$ is said to have a power-normal distribution with parameters $\lambda_{i}^{+}$and $\lambda_{i}^{-}([15])$.

When the values of the initial $k-1$ components of $\boldsymbol{y}$ are given, it is possible to find numerically the conditional distribution of the last component $y_{k}$ as demonstrated in [14].

\section{Probability of positive gain two months later}

The asset pricing model used in this paper involves a set of 17 selected monthly Malaysian microeconomic variables, the value and the volume of the Malaysian composite indices, and the prices and volumes of a number of pairs of selected Malaysian stocks.

The data set for the macroeconomic variables is obtained from the Statistics Department of the Central Bank of Malaysia. The 17 selected macroeconomic variables used in this study are listed in Table 1. These variables are found in the literature to have possible predictive power for the stock returns. The data for the chosen macroeconomic variables cover the period from January 2006 to December 2012. The timespan of the data is sufficient for the construction of model and the subsequent testing of the constructed model.

Table 1. Malaysian macroeconomic variables.

\begin{tabular}{|c|c|}
\hline $\begin{array}{c}\text { Assigned } \\
\text { Number }\end{array}$ & Macroeconomic Variable \\
\hline 1 & Gross domestic product (GDP) \\
\hline 2 & Producer price indicator \\
\hline 3 & Industrial production index \\
\hline 4 & Gross domestic savings \\
\hline 5 & Unemployment rate \\
\hline 6 & Interbank rate \\
\hline 7 & Money supply M2 \\
\hline 8 & Money supply M3 \\
\hline 9 & Total reserve money \\
\hline
\end{tabular}

\begin{tabular}{|c|c|}
\hline $\begin{array}{c}\text { Assigned } \\
\text { Number }\end{array}$ & Macroeconomic Variable \\
\hline 10 & Market indicative yield \\
\hline 11 & $\begin{array}{c}\text { Average discount rate on } \\
\text { Treasury bills }\end{array}$ \\
\hline 12 & $\begin{array}{c}\text { Consumer price index } \\
\text { (inflation) }\end{array}$ \\
\hline 13 & Oil price \\
\hline 14 & Gold price \\
\hline 15 & Trade (export) \\
\hline 16 & Trade (import) \\
\hline 17 & $\begin{array}{c}\text { Foreign exchange rate } \\
\text { (RM/USD) }\end{array}$ \\
\hline
\end{tabular}

Using the Malaysian macroeconomic data, a table (denoted as Table A) consisting of $T=$ 84 rows may be formed such that in the table, the $j$-th $(j=1,2, \ldots, T)$ row represents the values of the 17 monthly Malaysian macroeconomic variables when the $j$-th month is the current month. We form the $j_{w}$ th $\left(j_{w}=1,2, \ldots, T-n_{w}\right)$ sub-table of size $n_{w}+1$ using the 
$j_{w}$-th to $n_{w}+j_{w}$ rows of the table. Suppose $\boldsymbol{y}^{(1)}$ denotes the vector of 17 macroeconomic variables and each row of the sub-table represents an observed value of $\boldsymbol{y}^{(1)}$. The vector $\boldsymbol{y}^{(1)}$ may be described by a factor model with its static representation given by

$$
\boldsymbol{y}^{(1)}=\boldsymbol{A} \boldsymbol{F}+\boldsymbol{e}
$$

where $\boldsymbol{A}$ is a $17 \times r$ matrix of factor loadings, $\boldsymbol{F}$ is an $r \times 1$ vector of common latent factors underlying $\boldsymbol{y}^{(1)}$, and $\boldsymbol{e}$ is a $17 \times 1$ vector of random errors.

The principal component analysis is performed on the 17 columns of values in the subtable. Suppose the principal component with the $i$-th largest variance is denoted by $f_{i}$. The first $n_{L}$ principal components $\left(n_{L}<17\right)$ denoted by $f_{1}, f_{2}, \ldots, f_{n_{L}}$ are obtained. Let $f_{i j}$ be the value of $f_{i}$ extracted from the $j$-th row of the sub-table.

From the monthly data in the Malaysian stock market over the period January 2006 to December 2012, we can obtained a table (denoted as Table B) of 83 (when $k=1$ ) or 82 (when $k=2$ ) rows of length 7 with each row containing

(i) the trading volume in the Malaysian stock market in the present month,

(ii) the composite index of the Malaysian stock market in the present month,

(iii) the prices $S_{1}$ and $S_{2}$ the two selected stocks in the present month,

(iv) the trading volumes, $V_{1}$ and $V_{2}$ of the two selected stocks in the present month, and

(v) the next $k$-th month's price $S^{(k)}=w_{1} S_{1}^{(k)}+w_{2} S_{2}^{(k)}$ of the portfolio consisting of $w_{i}$ units of stock $i$ with $w_{1}+w_{2}=1$, for $i=1,2$ and $k=1,2$.

We form the $j_{w}$-th window of size $n_{w}+1$ by combining

(i) the values of the first $n_{L}$ principal components derived from the $j_{w}$-th sub-table of Table A and,

(ii) the $j_{w}$-th to $\left(n_{w}+j_{w}\right)$-th rows of Table $\mathrm{B}$.

A row in the $j_{w}$-th window may be considered as an observed value of a vector $\boldsymbol{y}$ of $n_{L}+7$ random variables. We fit a multivariate power-normal distribution [15] to the data given by the initial $n_{w}$ rows in the $j_{w}$-th window.

Given the values of the first $n_{L}+6$ components in the last row in the $j_{w}$-th window, we find a conditional distribution for the last component of the vector $y$. Let $S^{\left(j_{w}\right)(0)}$ be the observed value of the portfolio in the present month, $S^{\left(j_{w}\right)(1)}$ be the observed value of the portfolio next month and $g_{j_{w}}^{(k)}=\left(S^{(k)}-S^{\left(j_{w}\right)(0)}\right)$ the gain (before taking into account the interest rate and other expenses) in the next $k$-th month. We next compute

(i) the probability that the gain in the next $k$-th month is positive

(ii) The actual gain next month

$$
P_{j_{w}}^{(k)}=\mathbb{P}\left(g_{j_{w}}^{(k)}>0\right), \quad k=1,2 .
$$

$$
g_{j_{w}}^{(1 *)}=\left(S^{\left(j_{w}\right)(1)}-S^{\left(j_{w}\right)(0)}\right) .
$$

The value of next month actual gain $g_{j_{w}}^{(1 *)}$ may be regressed on $P_{j_{w}}^{(k)}$, for $k=1,2$. If the resulting regression coefficient is positive, and the value of $g_{j_{w}}^{(1 *)}$ tends to be positive when $P_{j_{w}}^{(k)}$ is large (for example $\geq 0.8$ ), then the probability $P_{j_{w}}^{(k)}, k=1,2$, may be used as an indicator for trading the portfolio.

\section{Numerical results}

This section will make use of the prices and trading volumes of the following six selected companies listed in Table 2 over the period January 2006 - December 2012. The stocks in Table 2 are selected such that the stocks cover some of the important sectors in the Malaysian stock market, and the stocks selected have complete data over the chosen period of study. 
Unlike data for the stock prices and volumes, the data for some of the Malaysian macroeconomic variables are not readily available. The data for the macroeconomic variables which have been obtained cover the period January 2006 to December 2012. In order to be able make use of the data on macroeconomic variables, the data for the stock prices and volumes are chosen to cover the same period.

Table 2. Six selected companies in Malaysian stock market.

\begin{tabular}{|c|c|c|}
\hline No. & Sector & Company Name \\
\hline 1 & Properties & Amcorp Properties Bhd \\
\hline 2 & Consumer Products & British American Tobacco Malaysia Bhd \\
\hline 3 & Industrial Products & DRB-HICOM Bhd \\
\hline 4 & Plantations & Golden Land Bhd \\
\hline 5 & Hotels & Landmarks Bhd \\
\hline 6 & Trading and Services & Star Media Group Bhd \\
\hline
\end{tabular}

We choose $n_{L}$ to be 2 and use the method in Section 3 to compute the probability $P_{j_{w}}^{(k)}$ for the value of $w_{1}$ which is varied from 0 to 1 with a step size of 0.05 .

Table 3 shows the maximum values of the slope $\hat{\beta}_{1}$ over the 21 chosen values of $w_{1}$, and the corresponding intercept $\hat{\beta}_{0}$ of the regression of $g_{j_{w}}^{(1 *)}$ on $P_{j_{w}}^{(k)}, k=1,2$, for the 15 possible pairs of stocks.

From Table 3, we see that the maximum value of the slope of the regression of the actual gain on the probability of positive gain in two months in the future tends to be larger than that of the regression on the probability of positive gain in the next month. For some pairs of stocks, the difference between the two maximum slopes can be fairly large.

Fig. 1 to Fig. 4 show the scatter diagrams of $g_{j_{w}}^{(1 *)}$ against $P_{j_{w}}^{(2)}$ when the pairs $(1,2),(1,4)$, $(2,6)$, and $(3,4)$ are used respectively, using the weights which yield the largest positive slope. These figures show that the actual gain $g_{j_{w}}^{(1 *)}$ next month tends to be positive when the probability $P_{j_{w}}^{(2)}$ of getting positive gain two months later is large (for example $\geq 0.8$ ). 
Table 3. The maximum values of the slope $\hat{\beta}_{1}$ and the corresponding intercept $\hat{\beta}_{0}$ of the regression of $g_{j_{w}}^{(1 *)}$ on $P_{j_{w}}^{(k)}, k=1,2$, for the 15 possible pairs of stocks.

\begin{tabular}{|c|c|r|r|r|r|r|r|r|r|}
\hline Stock & Stock & \multicolumn{4}{|c|}{ 1 month ahead } & \multicolumn{4}{|c|}{ 2 months ahead } \\
\cline { 3 - 9 } & $\mathbf{2}$ & $\boldsymbol{w}_{\mathbf{1}}$ & $\boldsymbol{w}_{\mathbf{2}}$ & \multicolumn{1}{c|}{$\widehat{\boldsymbol{\beta}}_{\mathbf{0}}$} & \multicolumn{1}{c|}{$\widehat{\boldsymbol{\beta}}_{\mathbf{1}}$} & $\boldsymbol{w}_{\mathbf{1}}$ & $\boldsymbol{w}_{\mathbf{2}}$ & \multicolumn{1}{c|}{$\widehat{\boldsymbol{\beta}}_{\mathbf{0}}$} & $\widehat{\boldsymbol{\beta}}_{\mathbf{1}}$ \\
\hline 1 & 2 & 0.70 & 0.30 & 0.129167 & 0.127135 & 0.05 & 0.95 & 0.531350 & 1.740048 \\
\hline 1 & 3 & 0.15 & 0.85 & -0.052213 & 0.166358 & 0 & 1 & -0.067865 & 0.178077 \\
\hline 1 & 4 & 0.45 & 0.55 & 0.001897 & 0.005050 & 0.70 & 0.30 & -0.008574 & 0.024656 \\
\hline 1 & 5 & 0.15 & 0.85 & -0.020444 & 0.031642 & 0.05 & 0.95 & -0.033034 & 0.069136 \\
\hline 1 & 6 & 1 & 0 & -0.010384 & 0.033370 & 1 & 0 & -0.012389 & 0.037079 \\
\hline 2 & 3 & 0.35 & 0.65 & 0.124982 & 0.286099 & 1 & 0 & -0.409748 & 1.625648 \\
\hline 2 & 4 & 0 & 1 & -0.000160 & 0.009869 & 1 & 0 & -0.410528 & 1.604899 \\
\hline 2 & 5 & 0 & 1 & -0.046669 & 0.122417 & 1 & 0 & -0.272103 & 1.436412 \\
\hline 2 & 6 & 0.40 & 0.60 & 0.309163 & -0.068934 & 1 & 0 & -0.469938 & 1.771072 \\
\hline 3 & 4 & 1 & 0 & -0.031461 & 0.154582 & 1 & 0 & -0.079393 & 0.201679 \\
\hline 3 & 5 & 1 & 0 & -0.038477 & 0.176892 & 1 & 0 & -0.076173 & 0.220547 \\
\hline 3 & 6 & 1 & 0 & -0.050652 & 0.188010 & 1 & 0 & -0.044550 & 0.160331 \\
\hline 4 & 5 & 0 & 1 & -0.043417 & 0.088234 & 0 & 1 & -0.047622 & 0.112152 \\
\hline 4 & 6 & 0.70 & 0.30 & 0.035313 & -0.046669 & 0 & 1 & 0.017320 & -0.019775 \\
\hline 5 & 6 & 1 & 0 & -0.062188 & 0.127752 & 1 & 0 & -0.074768 & 0.206272 \\
\hline
\end{tabular}

Stocks 1 and 2

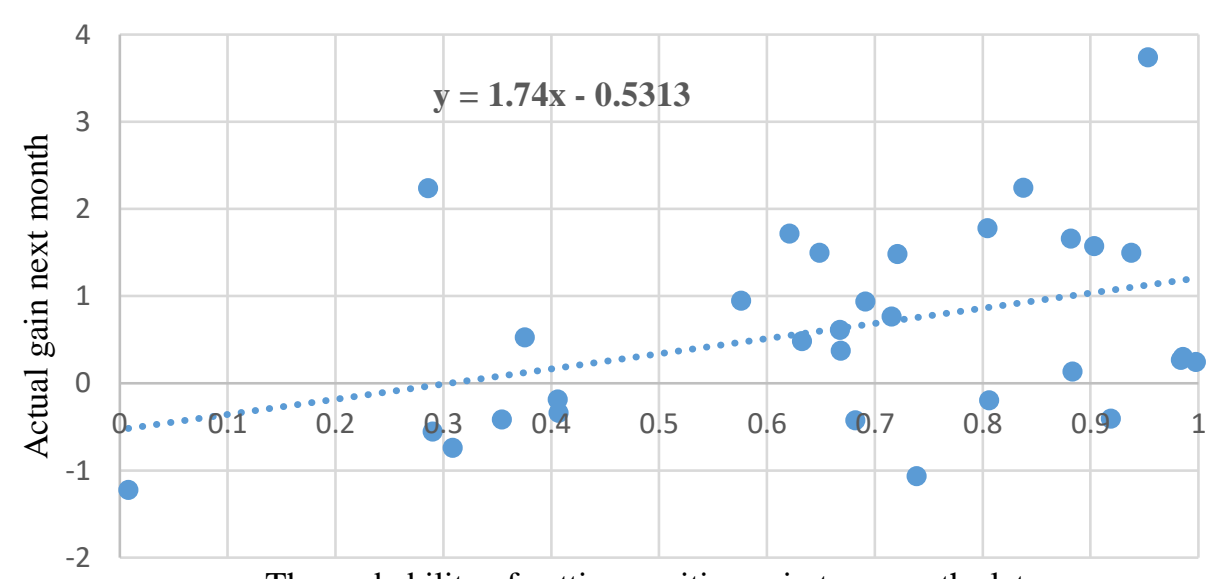

The probability of getting positive gain two months later

Fig. 1. The results of the regression of actual gain next month on the probability of getting positive gain two months later when stocks 1 and 2 are used. 


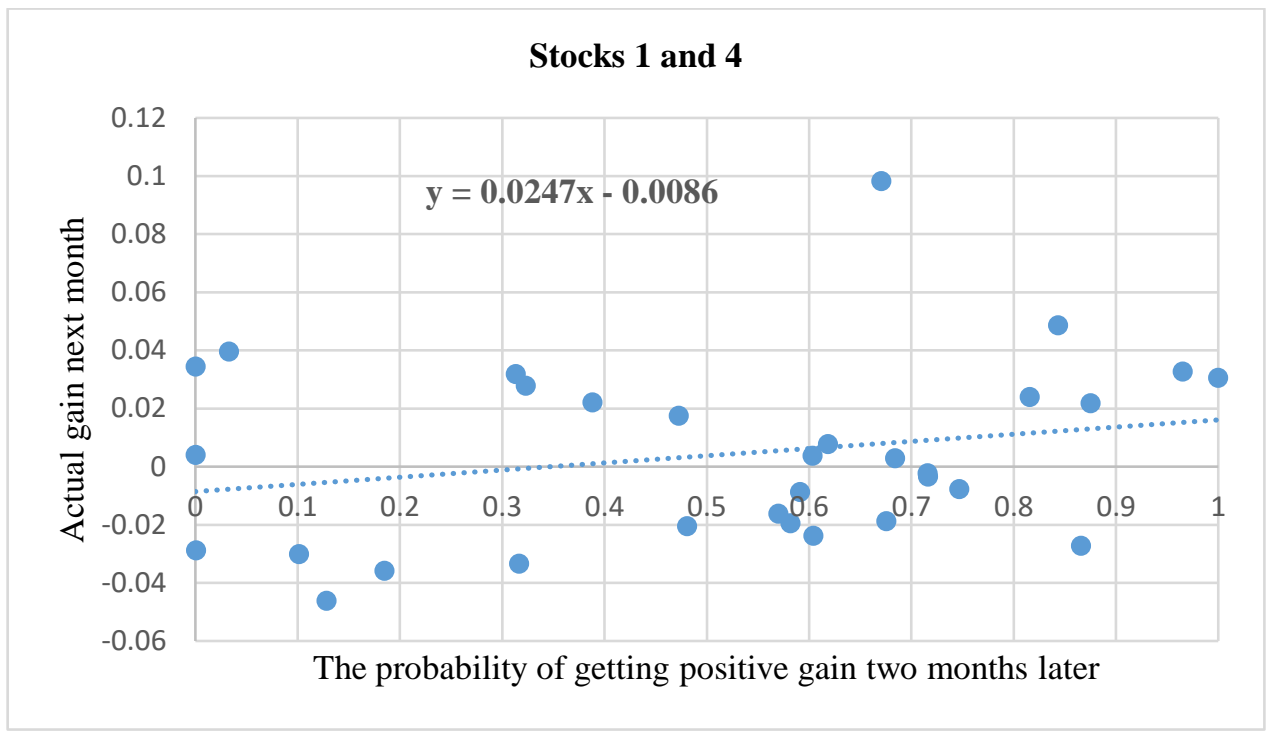

Fig. 2. The results of the regression of actual gain next month on the probability of getting positive gain two months later when stocks 1 and 4 are used.

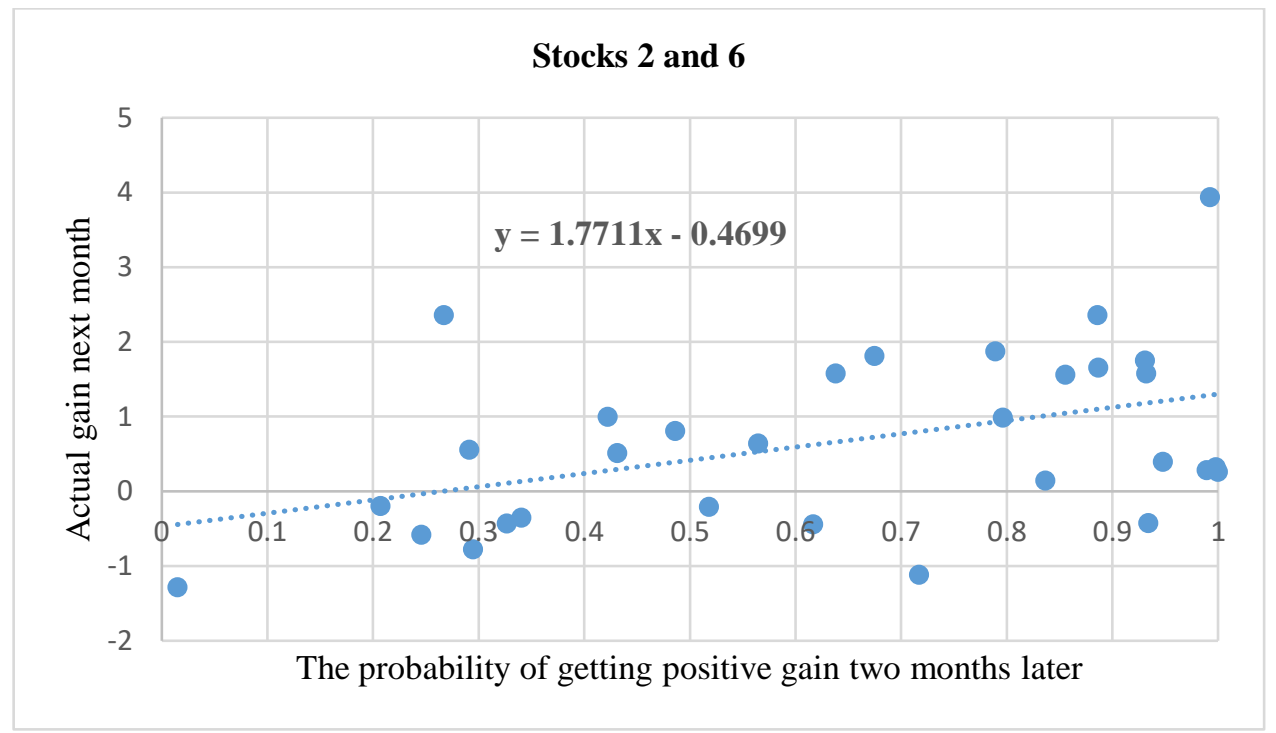

Fig. 3. The results of the regression of actual gain next month on the probability of getting positive gain two months later when stocks 2 and 6 are used. 


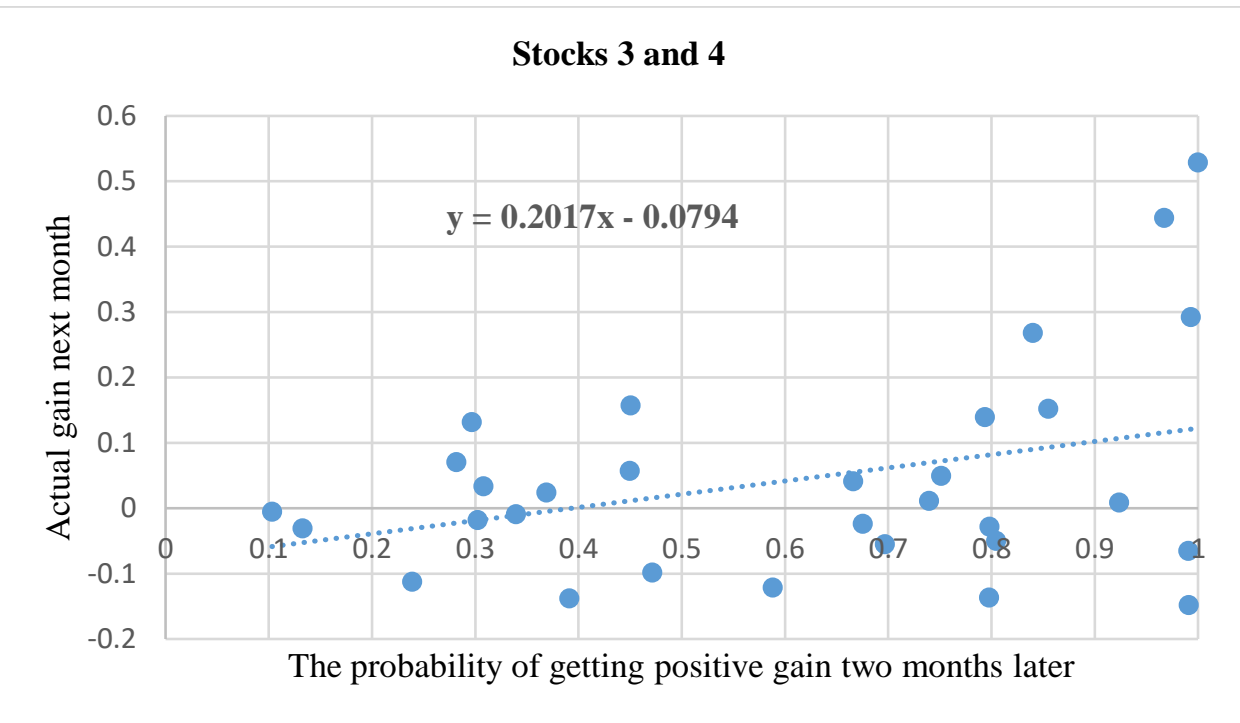

Fig. 4. The results of the regression of actual gain next month on the probability of getting positive gain two months later when stocks 3 and 4 are used.

\section{Concluding remarks}

The numerical results show that the proposed indicator works well for the portfolios of a number of pairs of Malaysian stocks. This indicates the predictability of some of the Malaysian monthly stock returns via the proposed model. The predictive power of the proposed model may be attributed to the incorporation of latent variables which extract the explanatory power of a large number of macroeconomic variables. The period of the data used is from Jan. 2006 to Dec. 2012. Although the proposed indicator works well for the data used, it is advisable to verify the usefulness of the indicator for the more recent data. It would be interesting to find out whether the indicator will work well also for the stocks in other markets. The case of portfolio of three or more stocks is another area for future research. However, a bigger challenge is the construction of a reliable model for daily stock returns and the subsequent formulation of a good indicator for day trading of stocks.

\section{References}

1. G. Appel, Technical analysis: power tools for active investors (Pearson Education, Upper Saddle River, 2005)

2. J.W. Wilder, New concepts in technical trading systems (Hunter Publishing Company, Winston-Salem, 1978)

3. J.E. Granville, Granville's new strategy of daily stock market timing for maximum profit (Prentice-Hall, Englewood Cliffs, 1976)

4. M.H. Pesaran, A. Timmermann, J. Finance 50, 1201 (1995)

5. A. Kyereboah-Coleman, K.F. Agyire-Tettey, J. Risk Finance 9, 365 (2008)

6. C. Cakmakli, D. van Dijk, Int. J. Forecast. 32, 650 (2016)

7. J. Liu, A. Kemp, Energy Econ. 81, 672 (2019)

8. M.A.B.M.T. Thaker, W. Rohilina, A. Hassama, M.F.B. Amin, Glob. J. Finance Econ. 7, 149 (2010) 
9. A.A. Rahman, N.Z. M. Sidek, F.H. Tafri, Afr. J. Bus. Manag. 3, 95 (2009)

10. Z. Zakaria, S. Shamsuddin, JBSQ 4, 61 (2012)

11. H.A. Bekhet, M.I. Mugableh, IJEF 4, 69 (2012)

12. F.A.M. Nasser, C.M.A.C. Rosid, N.S. Baharuddin, TIFEJ 5, 7 (2017)

13. Y.S. Ng, Modelling of asset prices using multivariate power-normal distributions, Ph.D (Business) thesis, Sunway University, Malaysia (2019)

14. A.H. Pooi, Appl. Math. Sci. 6, 5735 (2012)

15. I.K. Yeo, R. A. Johnson, Biometrika 87, 954 (2000) 